\title{
Manejo Nutricional em Paciente com Metástase Gástrica de Câncer de Mama: um Relato de Caso
}

\author{
Nutritional Management in a Patient with Breast Cancer Metastasis to the Stomach: a Case Report \\ Manejo Nutricional en Paciente con Metástasis Gástrica del Cáncer de Mama: un Informe de Caso
}

Larissa Calixto Lima'; Aline Pereira Pedrosa ${ }^{2}$; Fernanda de Oliveira Pereira ${ }^{3}$; Taiara Scopel Poltronieri ${ }^{4}$

Resumo

Introduçáo: O surgimento de metástase gástrica oriunda de neoplasia da mama é raro. Entretanto, o suporte nutricional nesses casos é imprescindível, já que tumores gástricos estão relacionados a complicaçôes nutricionais, favorecendo desfechos clínicos negativos. Relato do caso: Paciente com diagnóstico de adenocarcinoma de mama, com progressão para ossos, ovário e estômago, internada em razão das complicações clínicas. Apresentou perda de peso grave durante a internação, além de diagnóstico de risco de desnutrição pela avaliação subjetiva global produzida pelo paciente. Em virtude do quadro de odinofagia severa, a alimentaçáo via oral tornou-se impossibilitada, sendo optado pelo uso de sonda nasoentérica após discussão com equipe interdisciplinar. Conclusáo: A terapia nutricional em pacientes com câncer avançado ainda é um dilema entre os profissionais e sua execução deve ser discutida e decidida por uma equipe interdisciplinar, com participação indispensável do nutricionista, do paciente e de seus familiares. Para isso, os serviços de saúde devem estar preparados para o correto manejo da conduta nutricional, a fim de promover qualidade de vida para esses pacientes.

Palavras-chave: Neoplasias da Mama; Metástase Neoplásica; Terapia Nutricional.

\begin{abstract}
Introduction: It is uncommon for breast cancer to metastasize to the stomach. However, when it does occur, nutritional support is indispensable, because gastric tumors are associated with nutritional complications, which worsen clinical outcomes. Case report: We report the case of a female patient diagnosed with breast adenocarcinoma that had metastasized to the bones, ovary, and stomach, who was hospitalized because of clinical complications. During hospitalization, she presented severe weight loss and was classified as being at nutritional risk, as determined by the Patient-Generated Subjective Global Assessment. Due to severe odynophagia, oral intake was not possible. Therefore, after consultation with the multidisciplinary team, the decision was made to insert a nasogastric tube. Conclusion: Nutritional therapy in patients with advanced cancer still constitutes a dilemma facing professionals and needs to be discussed by a multidisciplinary team, the participation of the nutritionist, patient, and family being indispensable. Therefore, health care services must be prepared to manage nutritional therapy correctly, in order to promote better quality of life for such patients.

Key words: Breast Neoplasms; Neoplasm Metastasis; Nutrition Therapy.
\end{abstract}

Resumen

Introducción: El surgimiento de metástasis gástrica proveniente de neoplasia de mama es raro. Sin embargo, el soporte nutricional en estos casos es imprescindible, ya que los tumores gástricos están relacionados a complicaciones nutricionales, favoreciendo los resultados clínicos negativos. Informe de caso: Paciente con diagnóstico de adenocarcinoma de mama, con progresión para huesos, ovario y estómago, internada debido a complicaciones clínicas. Se presentó una pérdida de peso grave durante la internación, además de diagnóstico de riesgo de desnutrición por la evaluación subjetiva global producida por el paciente. Debido a un cuadro de odinofagia severa, la alimentación oral se volvió imposibilitada, siendo optado por el uso de sonda nasoentérica después de la discusión con el equipo interdisciplinario. Conclusión: La terapia nutricional en pacientes con cáncer avanzado sigue siendo un dilema entre los profesionales y su ejecución debe ser discutida y decidida por un equipo interdisciplinario, con participación indispensable del nutricionista, del paciente y de sus familiares. Para ello, los servicios de salud deben estar preparados para el correcto manejo de la conducta nutricional, a fin de promover calidad de vida para estos pacientes.

Palabras clave: Neoplasias de la Mama; Metástasis de la Neoplasia; Terapia Nutricional.

\footnotetext{
${ }^{1}$ Nutricionista. Mestre em Nutrição pelo Instituto de Nutrição da Universidade do Estado do Rio de Janeiro (Uerj). Hospital do Câncer IV (HC IV). Instituto Nacional de Câncer José Alencar Gomes da Silva (INCA). Rio de Janeiro (RJ), Brasil. E-mail: larissa.lima@inca.gov.br.

${ }^{2}$ Nutricionista. Graduação em Nutrição pela Universidade Federal do Rio de Janeiro (UFRJ). Discente do Programa de Residência Multiprofissional em Oncologia do INCA. Rio de Janeiro (RJ), Brasil. E-mail: alinepp.nut@gmail.com.

${ }^{3}$ Nutricionista. Graduação em Nutrição pela Universidade Federal do Estado do Rio de Janeiro (Unirio). Discente do Programa de Residência Multiprofissional em Oncologia do INCA. Rio de Janeiro (RJ), Brasil.E-mail: feoliveirananda@gmail.com.

${ }^{4}$ Nutricionista. Graduação em Nutrição pelo Centro Universitário da Serra Gaúcha (FSG). Discente do Programa de Residência Multiprofissional em Oncologia do INCA. Rio de Janeiro (RJ), Brasil.E-mail: taiarapoltronieri@hotmail.com.
}

Endereço para correspondência: Larissa Calixto Lima.E-mail: larissa.lima@inca.gov.br. 


\section{INTRODUÇÃO}

O câncer caracteriza-se pelo crescimento desordenado de células, podendo haver metástases para outros tecidos e órgãos ${ }^{1}$. Segundo o Instituto Nacional de Câncer José Alencar Gomes da Silva (INCA), a neoplasia de mama é a mais comum entre mulheres, e possui a maior incidência e mortalidade em todo o mundo ${ }^{2}$.

Tumores de mama metastáticos geralmente migram para ossos, pulmão, fígado, cérebro e linfonodos ${ }^{3}$. Entretanto, os novos tratamentos estão expandindo as taxas de sobrevivência, fazendo com que seja necessário atentar para o surgimento de progressôes em locais antes inesperados ${ }^{4}$.

De acordo com a literatura, a incidência de metástase gástrica oriunda do tumor de mama é de $1 \%$ ou menos ${ }^{3}$. Esse sítio metastático, quando presente, é quase sempre diagnosticado em estágios mais avançados, momento em que as terapêuticas, capazes de modificar o curso da doença, perdem efetividade e o cuidado paliativo ganha maior importância ${ }^{4}$. Dessa maneira, o término de uma terapia com proposta curativa não significa o final de um tratamento ativo, mas mudanças no foco da intervenção, cujo propósito principal é reduzir o sofrimento e fornecer melhor qualidade de vida ao indivíduo e sua família. Nos cuidados paliativos, os profissionais devem basear suas decisóes nas preferências e expectativas dos pacientes e familiares e, também, na avaliação criteriosa do prognóstico e da sobrevida. Uma avaliação adequada conduz a melhoria das estratégias de tratamento, subsidia o planejamento dos cuidados e a utilizaçáo eficiente dos recursos disponíveis, ajudando a minimizar os riscos de subtratamento ou de tratamentos excessivos e fúteis ${ }^{5}$.

Nesse contexto, diversas medidas de avaliação nutricional vêm sendo utilizadas como marcadores prognósticos em pacientes com tumores avançados, visto que indivíduos malnutridos têm maior risco de desfechos clínicos adversos quando comparados com aqueles bem-nutridos ${ }^{6}$. A intervenção nutricional é imprescindível e tem objetivos distintos de acordo com a conduta clínica para a qual o paciente será submetido, podendo atuar no controle de sintomas, melhorando a funcionalidade $\mathrm{e}$ proporcionando qualidade de vida ${ }^{7}$, até mesmo por meio da ressignificação do alimento, possibilitando a redução da ansiedade e maior prazer 5 .

Desse modo, o objetivo do presente estudo foi relatar o manejo nutricional realizado em uma paciente diagnosticada com câncer de mama avançado com metástase gástrica.

Foi realizado um estudo descritivo, do tipo relato de caso de experiência, sobre o cuidado nutricional a uma paciente diagnosticada com câncer de mama e metástase gástrica. Tal experiência ocorreu no Hospital do Câncer III (HCIII), do INCA (Rio de Janeiro, Brasil), unidade de referência para o tratamento do câncer de mama. O presente estudo foi submetido e aprovado pelo Comitê de Ética em Pesquisa da respectiva instituiçâo, sob o protocolo no 2.563.495 (CAAE: 83451518.5.0000.5274).

A avaliação do estado nutricional foi realizada de acordo com o protocolo de avaliação nutricional da unidade hospitalar em questão, que é composta pela Avaliação Subjetiva Global Produzida pelo Próprio Paciente (ASG-PPP), antropometria e parâmetros bioquímicos.

A ASG-PPP foi aplicada durante as primeiras 24 horas após internação hospitalar por um avaliador previamente treinado. Essa ferramenta, validada no Brasil por Gonzalez et al. ${ }^{8}$, avalia o estado nutricional a partir da combinação de fatores como perda de peso, alteraçóes na ingestão alimentar, sintomas gastrintestinais, alteraçóes funcionais e exame físico. A classificação do estado nutricional a partir da ASG-PPP é fornecida de forma subjetiva e dividida em três categorias: bem-nutrido (A); moderadamente desnutrido ou em risco de desnutrição (B); ou gravemente desnutrido (C).

A antropometria foi composta pela aferição do peso, estatura, dobra cutânea tricipital (DCT), circunferência do braço $(\mathrm{CB})$ e circunferência muscular do braço (CMB). O peso (em quilogramas) foi mensurado por meio de cama balança da marca Stryker com capacidade máxima para $228 \mathrm{~kg}$ e com precisão de $0,1 \mathrm{~kg}$. A estatura (em metros) foi referida pela paciente no momento da internação e confirmada nos registros anteriores em prontuário. $\mathrm{O}$ índice de massa corporal (IMC) foi obtido pela relação peso/(altura) ${ }^{2}$ e classificado conforme os pontos de corte propostos pela Organização Mundial da Saúde (OMS) ${ }^{9}$. A significância da perda de peso foi classificada conforme a classificação de Blackburn et al. ${ }^{10}$, a qual considera período e percentual de redução do peso.

A DCT foi verificada por meio de adipômetro Lange Skinfold Caliper, da marca TBW. A medida foi determinada no braço dominante, na face posterior, sendo seu ponto exato de reparo a distância entre o acrômio da escápula e o olecrano da ulna. Para a medição, o tecido gorduroso foi levemente desprendido do tecido muscular e pinçado com o calibrador, formando um ângulo reto exatamente no local marcado. Durante a avaliaçáo, o braço manteve-se relaxado e solto ao lado do corpo.

A CB foi realizada com utilização de uma fita métrica inextensível, no braço dominante, no mesmo ponto médio utilizado para aferir a DCT. Para a obtenção da $\mathrm{CB}$, o participante manteve o braço relaxado, permitindo que a fita contornasse o ponto marcado, de forma ajustada, porém evitando a compressão da pele. $\mathrm{A} \mathrm{CMB}$ 
foi obtida por meio da equação proposta por Gurney \& Jelliffe ${ }^{11}$, que emprega a CB e a DCT. A classificação do estado nutricional para $\mathrm{CB}, \mathrm{CMB}$ e DCT foi obtida ao se compararem os valores medidos com os valores de referência internacional (Percentil 50) do National Health and Nutrition Examination Survey, demonstrados em tabela de percentis por Frisancho ${ }^{12,13}$.

Os parâmetros bioquímicos avaliados foram hemograma completo, proteína $\mathrm{C}$ reativa (PCR) e albumina. Com os valores de PCR e albumina, foi calculado o escore prognóstico de Glasgow (EPG), definido como 2 quando as concentraçôes de PCR e albumina são, respectivamente, $<3,5 \mathrm{~g} / \mathrm{dL}$ e $>10 \mathrm{mg} / \mathrm{L}$ (pior prognóstico); como 1 quando as concentraçôes de albumina são $\geq 3,5 \mathrm{~g} / \mathrm{dL}$ e PCR $>10 \mathrm{mg} / \mathrm{L}$; e EPG igual a zero se $\mathrm{PCR} \leq 10 \mathrm{mg} / \mathrm{L}^{14}$.

\section{RELATO DO CASO}

Paciente do sexo feminino, 54 anos, branca, casada, com diabetes mellitus tipo II e hipertensa, em uso de insulina humana de forma regular conforme valor das glicemias e cloridrato de propranolol, respectivamente. Diagnosticada com carcinoma lobular invasivo (CLI) de mama esquerda, estádio IV (progressão para ossos, ovário e estômago), grau II, apresentando receptores hormonais (estrogênio e progesterona) positivos ( $>67 \%)$, HER-2 negativo, ki-67<14\%. Realizou radioterapia em quatro fraçōes por causa da síndrome de compressão medular e utilizou tamoxifeno por 30 dias. A paciente foi submetida à esofagogastroduodenoscopia com biópsia, onde foram evidenciadas lesóes no estômago, duas localizadas na grande curvatura do antro proximal e uma na parede posterior. A biópsia confirmou a presença de adenorcarcinoma gástrico, como metástase do tumor primário mamário. $\mathrm{O}$ estudo imuno-histoquímico revelou positividade para CK7 e para receptor de estrogênio. Em relação aos marcadores tumorais, encontraram-se resultados como aumento importante do antígeno carcinoembrionário (CEA) com valores de 170,50 ng/mL, CA-125 de 396,40 U/mL e CA 15,3 de $994,50 \mathrm{U} / \mathrm{mL}$.

Em razão da desidratação, infecção respiratória e candidíase oral, a paciente necessitou de internaçáo hospitalar. $\mathrm{Na}$ admissão hospitalar, foi realizada a triagem nutricional por meio da ASG-PPP. De acordo com essa ferramenta, a paciente foi classificada como moderadamente desnutrida ou em risco de desnutrição (ASG-PPP B, 19 pontos), indicando necessidade crítica de melhora no manuseio dos sintomas e/ou opçóes de intervenção nutricional.

$\mathrm{Na}$ avaliação antropométrica, também realizada no momento da internação, a paciente pesava $74,5 \mathrm{~kg}$, estatura de $1.57 \mathrm{~cm}$ e IMC indicativo de obesidade grau I $\left(30,2 \mathrm{~kg} / \mathrm{m}^{2}\right)$. Ademais, os percentis da DCT, CB e CMB eram adequados (CB e DCT estavam entre o percentil 50 e 75 e CMB entre o percentil 25 e 50). A paciente relatou peso de $80 \mathrm{~kg}$ um mês antes da realizaçáo da ASG-PPP e perda de peso de $2,5 \mathrm{~kg}(3,2 \%)$ em dez dias (Tabela 1$)$.

Após sete dias, foi realizada nova avaliação nutricional. Nesse momento, a paciente pesava $72,4 \mathrm{~kg}$, com IMC indicativo de sobrepeso $\left(29,4 \mathrm{~kg} / \mathrm{m}^{2}\right)$. Observou-se uma perda ponderal de $2,7 \%$ no período de uma semana, considerada perda de peso grave ${ }^{10}$. A paciente em questão apresentou perda de peso grave em todas as avaliaçóes nutricionais realizadas durante a internaçáo e perda de peso total de 9,4\% no período de um mês (Gráfico 1).

Os sintomas gastrintestinais relatados foram odinofagia, disfagia, xerostomia e constipação intestinal. Os exames laboratoriais evidenciaram hipoalbuminemia, plaquetopenia e anemia, além de funçóes renal e hepática preservadas. A relação entre os níveis séricos de PCR e albumina evidenciou EPG igual a zero (Tabela 1).

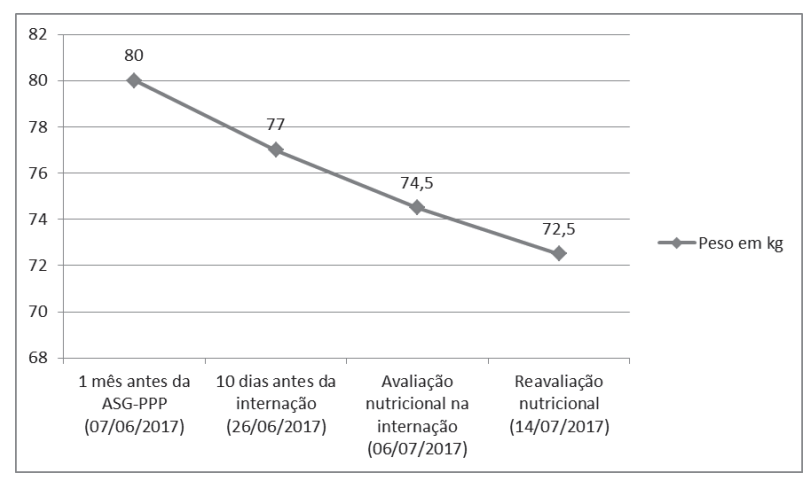

ASG-PPP: avaliação subjetiva global produzida pelo paciente; kg: quilogramas.

Gráfico 1. Acompanhamento da perda de peso da paciente em questão

De acordo Fearon ${ }^{15}$, a paciente em questão pode ser considerada como em pré-caquexia, visto que apresenta uma perda de peso significativa, perda de apetite e alteraçóes metabólicas visualizadas pelos resultados de hemograma e albumina. Levando em consideração as alteraçóes gastrointestinais e as comorbidades associadas, inicialmente a conduta nutricional foi uma nutrição via oral, consistência pastosa, fracionada em seis refeiçóes diárias, normocalórica, hiperproteica, hipossódica, isenta de alimentos irritantes da mucosa, restrita em carboidratos simples, laxativa e acrescida de suplementação nutricional via oral hipercalórica e hiperproteica, uma vez ao dia. A necessidade energética estimada foi de $2 \mathrm{mil} \mathrm{kcal} /$ dia $(25 \mathrm{kcal} / \mathrm{kg} / \mathrm{dia})$ e de proteína $120 \mathrm{~g} / \mathrm{dia}(1,5 \mathrm{~g} / \mathrm{kg} /$ dia), de acordo com as recomendaçóes do INCA ${ }^{14}$ para manutenção do peso em pacientes com estresse grave. 
Tabela 1. Avaliação antropométrica e bioquímica comparando os valores da admissão e da última avaliação nutricional da paciente com metástase gástrica de câncer mama

\begin{tabular}{|c|c|c|c|c|}
\hline $\begin{array}{l}\text { Parâmetros } \\
\text { antropométricos }\end{array}$ & $\begin{array}{l}\text { Admissão } \\
(6 / 7 / 2017)\end{array}$ & $\begin{array}{c}\text { Reavaliação } \\
\text { nutricional } \\
(14 / 7 / 2017)\end{array}$ & Classificação & Referências \\
\hline Peso (kg) & 74,5 & 72,5 & $* *$ & $* *$ \\
\hline Estatura (m) & 1,57 & 1,57 & $* *$ & $* *$ \\
\hline $\mathrm{IMC}\left(\mathrm{kg} / \mathrm{m}^{2}\right)$ & 30,2 & 29,4 & Sobrepeso & $\mathrm{OMS}^{9}$ \\
\hline PP (\%) & $* *$ & $\begin{array}{l}2,7 \% \text { em uma } \\
\text { semana }\end{array}$ & PP severa & Blackburn et al. ${ }^{10}$ \\
\hline CB $(\mathrm{cm})$ & $* *$ & 31,9 (p50-p75) & Adequado & Frisancho $^{13}$ \\
\hline $\mathrm{DCT}(\mathrm{mm})$ & $* *$ & 28 (p50-p75) & Adequado & Frisancho $^{13}$ \\
\hline $\mathrm{CMB}(\mathrm{cm})$ & $* *$ & $22,2(p 25-p 50)$ & Adequado & Frisancho $^{12}$ \\
\hline Dados bioquímicos & Admissão & $\begin{array}{c}\text { Reavaliação } \\
\text { nutricional }\end{array}$ & Classificação & Referências* \\
\hline Hematócrito (\%) & 38,5 & 25,9 & Baixo & $36-47$ \\
\hline Hemoglobina (g/dL) & 13,0 & 8,50 & Baixo & $11,5-16,4$ \\
\hline Leucócitos $(/ \mu \mathrm{l})$ & 6.900 & 5.200 & Adequado & $4.000-10.000$ \\
\hline Plaquetas $(\mathrm{k} / \mu \mathrm{l})$ & 140 & 93 & Baixo & $150-400$ \\
\hline PCR (mg/L) & Não solicitado & 8,64 & Alto & $<0,5$ \\
\hline Albumina (g/dL) & 3,4 & 2,4 & Baixo & $3,5-5,2$ \\
\hline EPG & $* *$ & 0 & Risco baixo & McMillan $^{14}$ \\
\hline
\end{tabular}

IMC: índice de massa corporal; PP: perda de peso; CB: circunferência do braço; DCT: dobra cutânea tricipital; CMB: circunferência muscular do braço; EPG: escore prognóstico de Glasgow; *Limites adotados pelo laboratório do Hospital do Câncer III/INCA; ${ }^{* *}$ Não se aplica.

A aceitação da dieta via oral era parcial. No decorrer da internaçáo, a paciente evoluiu com uma intensa dor em região torácica (esôfago) causando odinofagia, o que a levou a reduzir ainda mais a aceitaçáo da dieta oral ofertada. Nesse momento, optou-se pela passagem de sonda nasoentérica para início de nutrição enteral com dieta de sistema fechado, polimérica, hipercalórica, hiperproteica, isenta de sacarose, hipossódica, com gotejamento inicial de $25 \mathrm{ml} / \mathrm{h}$ e oferta de $750 \mathrm{kcal} /$ dia (37,5\% das necessidades energéticas diárias). No dia seguinte, houve evolução para $50 \mathrm{ml} / \mathrm{h}$ com um total de $1.500 \mathrm{kcal} / \mathrm{dia}$ ( $75 \%$ das necessidades energéticas diárias). No terceiro dia de dia de nutrição enteral, a paciente foi a óbito em razão da falência múltipla dos órgãos.

\section{DISCUSSÃO}

A metástase gástrica do câncer de mama, quando presente, é de difícil diagnóstico, já que os sinais clínicos são geralmente inespecíficos ou mesmo inexistentes ou ainda podem ser mascarados pelos efeitos colaterais oriundos do tratamento do tumor primário 4 . Um exemplo é o uso de terapia hormonal oral (como tamoxifeno), a qual pode desencadear efeitos colaterais gastrointestinais ${ }^{16}$. Maximizando o fato de que mulheres com câncer de mama metastático para estômago têm pior prognóstico ${ }^{4}$.

Entre os tipos tumorais encontrados no câncer de mama, o carcinoma ductal invasivo é o mais comum ${ }^{17}$.
No entanto, tende a progredir menos frequentemente para o trato gastrointestinal quando comparado ao $\mathrm{CLI}^{18}$.

Em relação ao estado nutricional, a paciente encontrava-se em risco nutricional segundo a ASG-PPP, embora apresentasse obesidade grau I segundo o IMC. A ASG-PPP é considerada o padrão-ouro para avaliação nutricional oncológica, uma vez que apresenta alta sensibilidade e especificidade para essa populaçãoo ${ }^{19}$. Assim, apesar de o IMC não ser indicativo de desnutrição, a perda de peso em pacientes oncológicos per se já é considerada como preocupante, pois provoca o aumento de complicaçóes e diminuição da sobrevida ${ }^{20}$. Esses dados enfatizam que, para a realização de uma avaliação nutricional adequada, com maior precisão e acurácia, tal avaliação deve ser composta pelo maior número de parâmetros possíveis, tais como medidas antropométricas, exames laboratoriais e funcionais do paciente e pelo olhar subjetivo do profissional ${ }^{20}$.

Nesse contexto, é imprescindível considerar a presença de caquexia - síndrome multifatorial caracterizada pela redução progressiva e involuntária do peso corporal e de músculo, podendo ou não haver perda de gordura, além de aumento do catabolismo proteico, da morbidade e mortalidade ${ }^{17}$. Essa condição é comum em pacientes com câncer avançado e afeta o desempenho e a qualidade de vida, estando associada a piores respostas ao tratamento. O consenso internacional para definição e classificação da caquexia do câncer divide a síndrome em três fases: a pré-caquexia, em que há perda de peso de até 5\% 
acompanhada de anorexia e alteraçóes metabólicas; a caquexia, em que há agravamento da perda de peso/massa muscular e o surgimento de inflamaçáo sistêmica; e a caquexia refratária, fase final da síndrome caracterizada por intenso catabolismo e não responsividade à terapia antitumoral, com baixa funcionalidade do paciente e expectativa de vida inferior a três meses ${ }^{17}$.

Visto que a inflamação sistêmica é característica do paciente oncológico, pode-se lançar mão de escores prognósticos para avaliação dessa condição, como o EPG, que tem se mostrado eficaz para essa população ${ }^{21}$. Dessa forma, a paciente em questão apresenta um EPG igual a zero, indicativo de melhor prognóstico. Esse escore utiliza como ponto de corte PCR $\geq 10 \mathrm{ml} / \mathrm{dL}$. Alguns estudos, no entanto, propóem pontos de corte ainda menores de PCR, de 7,5 mg/ $/ \mathrm{L}^{22}$ até mesmo $3,0 \mathrm{mg} / \mathrm{L}^{23}$, associados a piores desfechos clínicos.

A intervençâo nutricional éimprescindível, especificamente em casos de progressão neoplásica para o estômago, pois essa condição acarreta em inúmeras disfunçôes relacionadas à alimentação e à nutrição. No presente caso, a paciente manteve-se lúcida e orientada até a proximidade do seu fim de vida, além de dispor de acompanhante sempre presente, o qual se preocupava constantemente com o seu processo de alimentação, particularmente quando a alimentação via oral se tornou impossibilitada. Sendo assim, a escolha da via enteral para alimentação foi discutida pela equipe interdisciplinar, tendo como contexto uma paciente com impossibilidade de via oral pela odinofagia grave.

Em pacientes com câncer avançado, a decisão de iniciar a terapia nutricional enteral ou parenteral deve levar em consideração aspectos clínicos e prognósticos de forma individualizada, sobretudo as expectativas dos pacientes e familiares, já que os desejos e necessidades do paciente e cuidadores nessa fase são primordiais ${ }^{5,24}$. Porém, como a morte não é um evento probabilístico, seu tempo exato não pode ser descrito com $100 \%$ de certeza pelos fatores prognósticos ${ }^{25}$, o que pode levar os profissionais de saúde a terem condutas diferentes em relação a um mesmo caso.

\section{CONCLUSÃO}

A ocorrência de metástase gástrica em pacientes com câncer de mama é rara; porém, os serviços de saúde devem estar preparados para o correto manejo da conduta nutricional nesses casos, promovendo qualidade de vida - particularmente por meio do controle adequado dos sintomas - e evitando uma piora do estado nutricional, descontinuidade do tratamento e mortalidade precoce.

A ASG-PPP e a antropometria, mais especificamente a perda de peso, são importantes ferramentas que podem ser utilizadas como forma de diagnosticar a desnutrição. Ademais, o EPG pode ser utilizado como importante medida prognóstica. No entanto, é o olhar clínico e especializado que proporciona a melhor abordagem por toda a equipe, fato de grande relevância para o cuidado do paciente com câncer avançado. Dessa forma, levando em consideração que a administração da terapia nutricional em pacientes com câncer avançado ainda é um dilema entre os profissionais, a sua execução deve ser discutida e decidida por uma equipe interdisciplinar, com participação indispensável do nutricionista, do paciente e de seus familiares.

\section{CONTRIBUIÇÕES}

Aline Pereira Pedrosa, Fernanda de Oliveira Pereira e Taiara Scopel Poltronieri trabalharam em todas as etapas do estudo, desde a concepção até a versão final do artigo; ou seja, planejamento, obtenção e análise dos resultados e na redação e revisão crítica do texto. Larissa Calixto Lima trabalhou no auxílio à coleta dos dados, orientação da interpretação dos dados e na redação e revisão crítica do texto.

\section{DECLARAÇÃO DE CONFLITO DE INTERESSES}

Nada a Declarar.

\section{REFERÊNCIAS}

1. Brasil. Ministério da Saúde. Glossário temático controle de câncer: projeto de terminologia da saúde. Brasília: MS; 2003 [acesso em dez 2017]. Disponível em: $<$ http://bvsms.saude.gov.br/bvs/publicacoes/glossario_ tematico_controle_cancer.p>

2. Instituto Nacional de Câncer José Alencar Gomes da Silva. Estimativa 2018: incidência de câncer no Brasil. Rio de Janeiro: INCA; 2017 [acesso em mai 2018]. Disponível em: <http://www.inca.gov.br/ estimativa/2018/estimativa-2018.pdf>.

3. Yim K, Ro SM, Lee J. Breast cancer metastasizing to the stomach mimicking primary gastric cancer: a case report. World J Gastroenterol 2017;23(12):2251-7.

4. Fernandes GS, Faria LDBB, Pereira IA, Neves NCM, Vieira YO, Leal AIC. Gastric metastasis of breast cancer: a case series. Rare Tumors 2016;8(6305):94-6.

5. Instituto Nacional de Câncer José Alencar Gomes da Silva. Consenso nacional de nutriçáo oncológica. 2 ed. Rio de Janeiro: INCA; 2015 [acesso em dez 2017]. Disponível em: <http://www1.inca.gov.br/inca/ Arquivos/consensonacional-de-nutricao-oncologica-2edicao_2015_completo.pdf>. 
6. Choi WJ, Kim J. Nutritional care of gastric cancer patients with clinical outcomes and complications: a review. Clin Nutr Res 2016;5(2):65-78.

7. Birnstein E, Schattner M. Nutritional support in esophagogastric cancers. Surg Oncol Clin N Am 2017;26(2):325-33.

8. Gonzalez MC, Borges LR, Silveira DH, Assunção MCF, Orlandi SP. Validação da versão em português da avaliação subjetiva global produzida pelo paciente. Rev Bras Nutr Clin 2010;25(2):102-8.

9. World Health Organization (Switzerland). Obesity Presenting and managing the global epidemic: report of a WHO consultation. Geneva: WHO; 1998 [acesso em dez 2017]. Disponível em: <http://www.who.int/ nutrition/publications/obesity/WHO_TRS_894/en/>.

10. Blackburn GL, Bistrian BR, Maini BS, Schlamm, HT, Smith, MF. Nutritional and metabolic assessment of the hospitalized patient. JPEN, 1977; 1:11-32.

11. Gurney JM, Jelliffe DB. Arm anthropometry in nutritional assessment: nomogram for rapid calculation of muscle circunference and cross-sectional muscle and fat áreas. Am J Clin Nutr 1973;26(9):912-5.

12. Frisancho AR. New norms of upper limb fat and muscle areas for assessment of nutritional status. Am J Clin Nutr 1981;34(11):2540-5.

13. Frisancho AR. Anthropometric standards for the assessment of growth and nutritional status. Michigan: University of Michigan; 1990.

14. McMillan DC. An inflammation-based prognostic score and its role in the nutrition-based management of patients with cancer. Proc Nutr Soc 2008;67(3):257-62.

15. Fearon K, Strasser F, Anker SD, Bosaeus I, Bruera E, Fainsinger RL, et al. Definition and classification of cancer cachexia: an international consensus. Lancet Oncol 2011;12(5):489-95.

16. Wickersham K, Sereika SM, Bender CM. Pretreatment Predictors of Short-term Nonadherence to Oral Hormonal Therapy for Women with Breast Cancer. Nurs Res 2013;62(4):243-251.

17. Kita M, Furukawa M, Iwamuro M, Hori K, Kawahara Y, Taira N, et al. Breast cancer metastasis to the stomach that was diagnosed after endoscopic submucosal dissection. Case Rep Gastrointest Med 2016;2085452:1-5.

18. Ricciuti B, Leonardi GC, Ravaioli N, De Giglio A, Bramnilla M, Prosperi E, et al. Ductal breast carcinoma metastatic to the stomach resembling primary linitis plastica in a male patient. J Breast Cancer 2016;19(3):324-9.

19. Sociedade Brasileira de Nutrição Parenteral e Enteral/ Associação Brasileira de Nutrologia (Brasil). Terapia nutricional na oncologia. São Paulo (SP): Associação Médica Brasileira; 2011 [acesso em dez 2017]. Disponível em: <https://diretrizes.amb.org.br/_BibliotecaAntiga/ terapia_nutricional_na_oncologia.pdf>.
20. Cuppari L. Guias de medicina ambulatorial e hospitalar - Nutrição: nutrição clínica no adulto. 3 ed. São Paulo (SP): Manole; 2014. 578p.

21. Jin J, Hu K, Zhou Y, Li W. Clinical utility of the modified Glasgow prognostic score in lung cancer: a meta-analysis. PLoS ONE 2017;12(9):1-13.

22. Nakamura M, Iwahashi M, Nakamori M, Ojima T, Katsuda M, Iida T, et al. New prognostic score for the survival of patients with esophageal squamous cell carcinoma. Surg Today 2014;44:875-83.

23. Takeno S, Hashimoto T, Shibata R, Maki K, Shiwaku $\mathrm{H}$, Yamana I, et al. The high-sensitivity modified Glasgow prognostic score is superior to the modified Glasgow prognostic score as a prognostic predictor in patients with resectable gastric cancer. Oncology 2014;87(4):205-14.

24. Arends J, Baracos V, Bertz H, Bozzetti F, Calder PC, Deutz NEP, et al. ESPEN guidelines on nutrition in cancer patients. Clinical Nutrition, 2016.p. 1-38.

25. Bruera E, Hui D. Practical model for prognostication in advanced cancer patients: is less more? J Clin Oncol. 2008;26(36):5843-5844. 